\title{
High-precision limits on $W-W^{\prime}$ and $Z-Z^{\prime}$ mixing from diboson production using the full LHC Run 2 ATLAS data set
}

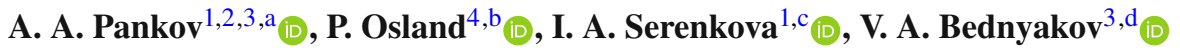 \\ ${ }^{1}$ The Abdus Salam ICTP Affiliated Centre, Technical University of Gomel, 246746 Gomel, Belarus \\ ${ }^{2}$ Institute for Nuclear Problems, Belarusian State University, 220030 Minsk, Belarus \\ ${ }^{3}$ Joint Institute for Nuclear Research, Dubna 141980, Russia \\ ${ }^{4}$ Department of Physics and Technology, University of Bergen, Postboks 7803, 5020 Bergen, Norway
}

Received: 10 December 2019 / Accepted: 23 May 2020 / Published online: 5 June 2020

(C) The Author(s) 2020

\begin{abstract}
The full ATLAS Run 2 data set with timeintegrated luminosity of $139 \mathrm{fb}^{-1}$ in the diboson channels in hadronic final states is used to probe a simple model with an extended gauge sector (EGM), proposed by Altarelli et al., and often taken as a convenient benchmark by experimentalists. This model accommodates new charged $W^{\prime}$ and neutral $Z^{\prime}$ vector bosons with modified trilinear Standard Model gauge couplings, decaying into electroweak gauge boson pairs $W Z$ or $W W$, where $W / Z$ decay hadronically. Exclusion limits at the $95 \% \mathrm{CL}$ on the $Z^{\prime}$ and $W^{\prime}$ resonance production cross section times branching ratio to electroweak gauge boson pairs in the mass range of $\sim 1-5 \mathrm{TeV}$ are here converted to constraints on $W-W^{\prime}$ and $Z-Z^{\prime}$ mixing parameters and masses for the EGM. We present exclusion regions on the parameter space of the $W^{\prime}$ and $Z^{\prime}$ by using the full Run 2 data set comprised of $p p$ collisions at $\sqrt{s}=13 \mathrm{TeV}$ and recorded by the ATLAS detector at the CERN LHC. The obtained exclusion regions are significantly extended compared to those obtained from the previous analysis performed with Tevatron data as well as with LHC data collected at 7 and $8 \mathrm{TeV}$ in Run 1 and are the most stringent bounds to date.
\end{abstract}

\section{Introduction}

One of the main aims of the physics programme at the Large Hadron Collider (LHC) is to search for new phenomena that become visible in high-energy proton-proton collisions. A possible signature of such new phenomena would be the production of a heavy resonance with its subsequent decay

\footnotetext{
a e-mail: pankov@ictp.it

be-mail: Per.Osland@uib.no (corresponding author)

c e-mail: Inna.Serenkova@cern.ch

de-mail: bedny@jinr.ru
}

into a final state consisting of a pair of fermions or vector bosons. Many new physics scenarios beyond the Standard Model (SM) predict such a signal. Possible candidates are charged and neutral heavy gauge bosons. In the simplest models these particles are considered copies of the SM $W$ and $Z$ bosons and are commonly referred to as $W^{\prime}$ and $Z^{\prime}$ bosons [1]. In the Sequential Standard Model (SSM) [2] the $W_{\mathrm{SSM}}^{\prime}$ and $Z_{\mathrm{SSM}}^{\prime}$ bosons have couplings to fermions that are identical to those of the SM $W$ and $Z$ bosons, but for which the trilinear couplings $W^{\prime} W Z$ and $Z^{\prime} W W$ are absent. The SSM has been used as a reference for experimental $W^{\prime}$ and $Z^{\prime}$ boson searches for decades, the results can be re-interpreted in the context of other models of new physics, and it is useful for comparing the sensitivity of different experiments.

At the LHC, such heavy $W^{\prime}$ and $Z^{\prime}$ bosons could be observed through their single production as $s$-channel resonances with subsequent leptonic decays

$p p \rightarrow W^{\prime} X \rightarrow \ell v X$,

and

$p p \rightarrow Z^{\prime} X \rightarrow \ell^{+} \ell^{-} X$,

respectively, where in what follows, $\ell=e, \mu$ unless otherwise stated. The production of $W^{\prime}$ and $Z^{\prime}$ bosons at hadron colliders is expected to be dominated by the process $q \bar{q}^{\prime} / q \bar{q} \rightarrow W^{\prime} / Z^{\prime}$. Leptonic final states provide a lowbackground and efficient experimental signature that results in excellent sensitivity to new phenomena at the LHC. Specifically, these processes (1.1) and (1.2) offer the simplest event topology for the discovery of $W^{\prime}$ and $Z^{\prime}$ with a large production rate and a clean experimental signature. These channels are among the most promising discoveries at the LHC [3-7]. There have also been many theoretical studies of $W^{\prime}$ and $Z^{\prime}$ boson searches at the high energy hadron colliders (see, e.g. $[1,8-25])$. 
The data we consider were collected with the ATLAS and CMS detectors during the 2015-2018 running period of the LHC, referred to as Run 2 and corresponding to timeintegrated luminosity of $139 \mathrm{fb}^{-1}$. The ATLAS experiment has presented the first search for dilepton resonances based on the full Run 2 data set $[3,7]$ and set limits on the $W^{\prime}$ and $Z^{\prime}$ production cross sections times branching fraction in the processes (1.1) and (1.2), $\sigma\left(p p \rightarrow W^{\prime} X\right) \times \mathrm{BR}\left(W^{\prime} \rightarrow \ell v\right)$ and $\sigma\left(p p \rightarrow Z^{\prime} X\right) \times \mathrm{BR}\left(Z^{\prime} \rightarrow \ell^{+} \ell^{-}\right)$, respectively, for $M_{W^{\prime}}$ and $M_{Z^{\prime}}$ in the $0.15-7 \mathrm{TeV}$ and $0.25-6 \mathrm{TeV}$ ranges, correspondingly. Recently, similar searches have also been presented by the CMS Collaboration using $140 \mathrm{fb}^{-1}$ of data recorded at $\sqrt{s}=13 \mathrm{TeV}$ [4]. The most stringent limits on the mass of $W_{\mathrm{SSM}}^{\prime}$ and $Z_{\mathrm{SSM}}^{\prime}$ bosons to date come from the searches in respectively, (1.1) and (1.2) processes by the ATLAS and CMS collaborations using data taken at $\sqrt{s}=13 \mathrm{TeV}$ in Run 2 and set a 95\% confidence level (CL) lower limit on the $W_{\mathrm{SSM}}^{\prime}$ mass of $6.0 \mathrm{TeV}[7]$ and $\sim 5.2 \mathrm{TeV}$ for $Z_{\text {SSM }}^{\prime}[3,4]$.

Alternative $W^{\prime}$ and $Z^{\prime}$ search channels are the diboson reactions

$p p \rightarrow W^{\prime} X \rightarrow W Z X$,

and

$p p \rightarrow Z^{\prime} X \rightarrow W W X$.

The study of gauge boson pair production offers a powerful test of the spontaneously broken gauge symmetry of the SM and can be used as a probe for new phenomena beyond the SM.

Heavy resonances that can decay to gauge boson pairs are predicted in many scenarios of new physics, including extended gauge models (EGM) [2,26], models of warped extra dimensions [27,28], technicolour models [29,30] associated with the existence of technirho and other technimesons, more generic composite Higgs models [31,32], and the heavy vector-triplet (HVT) model [33], which generalises a large number of models that predict spin-1 charged $\left(W^{\prime}\right)$ and neutral $\left(Z^{\prime}\right)$ resonances etc. Searches for exotic heavy particles that decay into $W Z$ or $W W$ pairs are complementary to searches in the leptonic channels $\ell v$ and $\ell^{+} \ell^{-}$of the processes (1.1) and (1.2). Moreover, there are models in which new gauge boson couplings to SM fermions are suppressed, giving rise to a fermiophobic $W^{\prime}$ and $Z^{\prime}$ with an enhanced coupling to electroweak gauge bosons [1,34]. It is therefore important to search for $W^{\prime}$ and $Z^{\prime}$ bosons also in the $W Z$ and $W W$ final states.

The paper is organized as follows. In Sect. 2 we present the theoretical framework, then, in Sect. 3 we summarize the relevant cross sections for processes (1.3) and (1.4) in the narrow width approximation (NWA) to the EGM. Next, in Sect. 4 we discuss the relevant $W^{\prime}$ and $Z^{\prime}$ branching ratios. In Sect. 5, we present an analysis of bounds on $W-W^{\prime}$ and $Z-Z^{\prime}$ mixing from constraints on diboson production in the context of the EGM, employing the most recent measurements recorded by the ATLAS $\left(36.7 \mathrm{fb}^{-1}\right.$ and $\left.139 \mathrm{fb}^{-1}\right)$ detector $[36,37]$ at the LHC. Then, we show the resulting constraints on the $M_{W^{\prime}}-\xi_{W-W^{\prime}}$ and $M_{Z^{\prime}}-\xi_{Z-Z^{\prime}}$ parameter space obtained from the diboson processes, (1.3) and (1.4). Further, we collect and compare the indirect constraints obtained from electroweak precision data, direct search constraints derived from the Tevatron and at the LHC in Run 1 and Run 2 data. Section 6 presents some concluding remarks.

\section{Framework}

Because of the large variety of models which predict new heavy charged and neutral gauge bosons, after a discovery of signatures associated to a new boson, detailed studies must be carried out to distinguish between these models and to determine whether the boson belongs to one of the theoretically motivated models such as, e.g. EGM or some other model. Following the traditions of direct searches at hadron colliders, such studies are based on the model first proposed in Ref. [2].

As mentioned above, in the SSM, the coupling constants of the $W^{\prime}$ and $Z^{\prime}$ bosons with SM fermions are identical to the corresponding SM couplings, while the $W^{\prime}$ and $Z^{\prime}$ couplings to, respectively, $W Z$ and $W W$ vanish, $g_{W^{\prime} W Z}=g_{Z^{\prime} W W}=$ 0 . Such a suppression may arise in an EGM in a natural manner: if the new gauge bosons and the SM ones belong to different gauge groups, vertices such as $W^{\prime} W Z$ and $Z^{\prime} W W$ do not arise. They can only occur after symmetry breaking due to mixing of the gauge eigenstates. Triple gauge boson couplings (such as $W^{\prime} W Z$ and $Z^{\prime} W W$ ) as well as the vectorvector-scalar couplings (like $W^{\prime} W H$ and $Z^{\prime} Z H$ ) arise from the symmetry breaking and may contribute to the $W^{\prime}$ and $Z^{\prime}$ decays, respectively. The vertices are then suppressed by a factor of the order of $\left(M_{W} / M_{V^{\prime}}\right)^{2}$, where $V^{\prime}$ represents a $W^{\prime}$ or a $Z^{\prime}$ boson.

In an EGM [2], the trilinear gauge boson couplings are modified by mixing factors

$\xi_{V-V^{\prime}}=\mathcal{C} \times\left(M_{W} / M_{V^{\prime}}\right)^{2}$,

where $\mathcal{C}$ is a scaling constant that sets the coupling strength. Specifically, in an EGM the standard-model trilinear gauge boson coupling strength $g_{W W Z}\left(=e \cot \theta_{W}\right)$, is replaced by $g_{W^{\prime} W Z}=\xi_{W-W^{\prime}} \cdot g_{W W Z}$ in the $W Z$ channel and $g_{Z^{\prime} W W}=\xi_{Z-Z^{\prime}} \cdot g_{W W Z}$ in the $W W$ channel. Following the parametrization of the trilinear gauge boson couplings $W^{\prime} W Z$ and $Z^{\prime} W W$ presented in [35] for the analysis and interpretation of the CDF data on $p \bar{p} \rightarrow W^{\prime} X \rightarrow W Z X$ and $p \bar{p} \rightarrow Z^{\prime} X \rightarrow W^{+} W^{-} X$, expressed in terms of two 
free parameters, ${ }^{1} \xi_{W-W^{\prime}}\left(\xi_{Z-Z^{\prime}}\right)$ and $M_{W^{\prime}}\left(M_{Z^{\prime}}\right)$, we will set $W^{\prime}\left(Z^{\prime}\right)$ limits as functions of the mass $M_{W^{\prime}}\left(M_{Z^{\prime}}\right)$ and mixing factor $\xi_{W-W^{\prime}}\left(\xi_{Z-Z^{\prime}}\right)$ by using the ATLAS resonant diboson production data $[36,37]$ collected at a center of mass energy of $\sqrt{s}=13 \mathrm{TeV}$, taking into account the partial and full Run 2 data sets with time-integrated luminosity of $36.7 \mathrm{fb}^{-1}$ and $139 \mathrm{fb}^{-1}$, respectively. The presented analysis in the EGM with two free parameters is more general than the previous ones where the only parameter is the $V^{\prime}$ mass. As for the SSM, one has $V_{\mathrm{SSM}}^{\prime} \equiv V_{\mathrm{EGM}}^{\prime}\left(\xi_{V-V^{\prime}}=0\right)$.

The parametrization of boson mixing introduced by Altarelli et al. [2], though being simplified, has a wellmotivated theoretical basis. To be specific, we briefly consider $Z^{0}-Z^{0 \prime}$ mixing within the framework of models with extended gauge sector such as the $E_{6}$ models, the LR model and SSM (see, e.g. [9,10,17-19]).

The physical (mass eigenstates) $Z$ and $Z^{\prime}$ are admixtures of the weak eigenstates $Z^{0}$ of $S U(2) \times U(1)$ and $Z^{0 \prime}$ of the extra $U(1)^{\prime}$, respectively. The mass eigenstates, $Z$ and $Z^{\prime}$ are obtained by a rotation of the fields $Z^{0}$ and $Z^{0 \prime}$ :

$$
\begin{aligned}
Z & =Z^{0} \cos \phi+Z^{0 \prime} \sin \phi, \\
Z^{\prime} & =-Z^{0} \sin \phi+Z^{0 \prime} \cos \phi .
\end{aligned}
$$

For each type of $Z^{\prime}$ boson, defined by each set of gauge couplings, there are three classes of models, which differ in the assumptions concerning the quantum numbers of the Higgs fields which generate the $Z$-boson mass matrix [9, $10,17]$. In each case there is a relation between the $Z^{0}-Z^{0 \prime}$ mixing angle $\phi$ and the two mass eigenvalues $M_{Z}$ and $M_{Z^{\prime}}$ which can be written as $[8,10]$ :

$\tan ^{2} \phi=\frac{M_{Z^{0}}^{2}-M_{Z}^{2}}{M_{Z^{\prime}}^{2}-M_{Z^{0}}^{2}}$,

where $M_{Z^{0}}$ is the mass of the $Z$ boson in the absence of mixing, i.e., for $\phi=0$. The mixing angle $\phi$ will play an important role in our analysis. Such mixing effects reflect the underlying gauge symmetry and/or the Higgs sector of the model:

(i) The least constrained ( $\rho_{0}$ free) model makes no assumption concerning the Higgs sector. It allows arbitrary $S U$ (2) representations for the Higgs fields, and is the analog of allowing $\rho_{0} \neq 1$ in the $S U(2) \times U(1)$ model. In this case $M_{Z}, M_{Z^{\prime}}$ and $\phi$ are all free parameters.

(ii) If one assume that all $S U(2)$ breaking is due to Higgs doublets and singlets ( $\rho_{0}=1$ model $)$, there are only two free parameters, which we identify as $\phi$ and $M_{Z^{\prime}}$, and we will adopt this parametrization throughout the paper, specifically for the EGM case.

\footnotetext{
${ }^{1}$ Such $W^{\prime}$ and $Z^{\prime}$, described in terms of two parameters, are here referred to as the EGM bosons.
}

(iii) Finally, in specific models one specifies not only the $S U(2)$ assignments but the $U(1)^{\prime}$ assignments of the Higgs fields. Since the same Higgs multiplets generate both $M_{Z}$ and $\phi$, one has an additional constraint. To a good approximation, for $M_{Z} \ll M_{Z^{\prime}}$, in specific "minimal-Higgs models", one has an additional constraint [8]

$$
\phi \simeq-s_{\mathrm{W}}^{2} \frac{\sum_{i}\left\langle\Phi_{i}\right\rangle^{2} I_{3 L}^{i} Q_{i}^{\prime}}{\sum_{i}\left\langle\Phi_{i}\right\rangle^{2}\left(I_{3 L}^{i}\right)^{2}}=P \frac{M_{Z}^{2}}{M_{Z^{\prime}}^{2}},
$$

where $s_{\mathrm{W}}$ is the sine of the electroweak angle. In these models $\phi$ and $M_{Z^{\prime}}$ are not independent and there is only one (e.g., $M_{Z^{\prime}}$ ) free parameter. This parametrization corresponds to the expression of the mixing factor presented in Eq. (2.1). Furthermore, $\left\langle\Phi_{i}\right\rangle$ are the Higgs (doublet) vacuum expectation values spontaneously breaking the symmetry, and $Q_{i}^{\prime}$ are their charges with respect to the additional $U(1)^{\prime}$. In these models the same Higgs multiplets are responsible for both generation of the mass $M_{Z}$ and for the strength of the $Z^{0}-Z^{0 \prime}$ mixing. Thus $P$ is a model-dependent constant.

This mixing between $Z^{0}$ and $Z^{0 \prime}$ will induce a change in couplings of the two bosons to fermions. An important property of the models under consideration is that the gauge eigenstate $Z^{0 \prime}$ does not couple to the $W^{+} W^{-}$pair since it is neutral under $S U(2)$. Therefore the $W$-pair production is sensitive to a $Z^{\prime}$ only in the case of a non-zero $Z^{0}-Z^{0 \prime}$ mixing. From (2.2) and (2.3), one obtains:

$$
\begin{aligned}
& g_{W W Z}=\cos \phi g_{W W Z^{0}}, \\
& g_{W W Z^{\prime}}=-\sin \phi g_{W W Z^{0},},
\end{aligned}
$$

where $g_{W W Z^{0}}=e \cot \theta_{W}$. Also, $g_{W W \gamma}=e$.

In many extended gauge models, while the couplings to fermions are not much different from those of the SM, the $Z^{\prime} W W$ coupling is substantially suppressed with respect to that of the SM. In fact, in the extended gauge models the SM trilinear gauge boson coupling strength, $g_{W W Z^{0}}$, is replaced by $g_{W W Z^{0}} \rightarrow \xi \cdot g_{W W Z^{0}}$, where $\xi \equiv|\sin \phi|$ (see Eq. (2.6b)) is the mixing factor. ${ }^{2}$ We will set cross section limits on such $Z^{\prime}$ as functions of the mass $M_{Z^{\prime}}$ and $\xi$.

Previous analyses of the $Z-Z^{\prime}$ and $W-W^{\prime}$ mixing [38$40]^{3}$ were carried out using the diboson production data set corresponding to the time-integrated luminosity of $\sim 36 \mathrm{fb}^{-1}$ collected in 2015 and 2016 with the ATLAS and CMS collaborations at $\sqrt{s}=13 \mathrm{TeV}$ where electroweak $Z$ and $W$ gauge bosons decay into the semileptonic channel [41] or into the

\footnotetext{
${ }^{2}$ For weak mixing, $\xi \simeq|\phi|$, and is therefore often referred to as a mixing "angle".

3 Strictly speaking, " $Z-Z^{\prime}$ mixing" should be referred to as " $Z^{0}-Z^{0 \prime}$ mixing" and similarly for " $W-W^{\prime}$ mixing".
} 
dijet final state [42]. The results of the present analysis benefit from the increased size of the data sample corresponding to an integrated luminosity of $139 \mathrm{fb}^{-1}$ recorded by the ATLAS detector in Run 2 [37] which is almost four times larger than what was available for the previous study in the semileptonic final state. ${ }^{4}$ In addition, further improvement in placing limits on the $W^{\prime}$ and $Z^{\prime}$ mass and $W-W^{\prime}$ and $Z-Z^{\prime}$ mixing parameters can be achieved in fully-hadronic $W Z / W W \rightarrow q q q q$ final states ${ }^{5}$ using the novel reconstruction and analysis techniques of a diboson system with pairs of large-radius jets. Indeed, the $W$ and $Z$ bosons produced in the decay of TeVscale resonances are highly energetic ("boosted") so that their decay products are merged into a single large-radius jet, and are therefore reconstructed experimentally as a single largeradius-parameter jet and accordingly, interpreted as a two-jet final state. The signature of such heavy resonance decays is thus a resonant structure in the dijet invariant mass spectrum. This novel technique allows to improve background estimation and the signal extraction procedure, resulting in higher sensitivity of the analysis.

The properties of possible $W^{\prime}$ and $Z^{\prime}$ bosons are also constrained by measurements of electroweak $(\mathrm{EW})$ processes at low energies, i.e., at energies much below the masses of new charged and neutral gauge bosons. Such bounds on the $W-$ $W^{\prime}\left(Z-Z^{\prime}\right)$ mixing are mostly due to the deviation in $W(Z)$ properties compared to the SM predictions. These measurements show that the mixing angles $\xi_{W-W^{\prime}}$ and $\xi_{Z-Z^{\prime}}$ between the gauge eigenstates must be smaller than about $10^{-2}$ and $2.6 \cdot 10^{-3}$, respectively $[1,17]$.

In this work, we derive bounds on the possible new spin1 resonances $\left(W^{\prime} / Z^{\prime}\right)$ within the EGM framework, from the full ATLAS Run 2 data set on $W Z / W W$ pair production with time-integrated luminosity of $139 \mathrm{fb}^{-1}$ [37]. The search was conducted for a $W^{\prime} / Z^{\prime}$ resonance decaying into a $W Z / W W$ boson pair, where the $W$ and $Z$ bosons decay hadronically. We present results as constraints on the relevant $W-W^{\prime}(Z-$ $\left.Z^{\prime}\right)$ mixing angle, $\xi_{W-W^{\prime}}\left(\xi_{Z-Z^{\prime}}\right)$, and on the mass $M_{W^{\prime}}$ $\left(M_{Z^{\prime}}\right)$ and display the combined allowed parameter space for the benchmark $W^{\prime}\left(Z^{\prime}\right)$ bosons, showing also indirect constraints from electroweak precision data, previous direct search constraints from the Tevatron and from the LHC with 7 and $8 \mathrm{TeV}$ in Run 1 as well as those obtained from the LHC at $13 \mathrm{TeV}$ with a partial ATLAS Run 2 data set with time integrated luminosity of $36.7 \mathrm{fb}^{-1}$ [36] in the fully hadronic (qqqq) final states.

Let us here comment on possible mechanisms that might generate the $V-V^{\prime}$ mixing. Within a UV-complete theory,

\footnotetext{
${ }^{4}$ In the current analysis, we utilize the full Run 2 ATLAS data set on diboson resonance production [37], rather than that of CMS, as the latter one is unavailable so far.

5 To simplify notation, antiparticles are denoted by the same symbol as the corresponding particles.
}

mixing could enter the trilinear coupling via the kinetic terms,

$$
-\frac{1}{2} \operatorname{Tr}\left[V_{\mu \nu}^{\dagger} V^{\mu \nu}\right], \quad \text { with } V_{\mu \nu}=\left[D_{\mu}, D_{\nu}\right],
$$

where the covariant derivative includes the heavier gauge field, $V_{\mu}^{\prime}$, schematically

$D_{\mu}=D_{\mu}^{\mathrm{SM}}+g^{\prime} V_{\mu}^{\prime}$.

An off-diagonal term in the mass-squared matrix would lead to mixing as given by Eq. (2.4). On the other hand, mixing could be a loop effect. While such examples of mechanisms do not offer much insight on the magnitude of the mixing, they would allow for an interpretation of an observed signal.

\section{Resonant diboson production in $p p$ collision}

At lowest order in the EGM, $W^{\prime}$ production and decay into $W Z$ in proton-proton collisions occurs through quarkantiquark interactions in the $s$-channel. The cross section of process (1.3) can at the LHC be observed through resonant pair production of gauge bosons $W Z$. Using the NWA, one can factorize the process (1.3) into the $W^{\prime}$ production and the $W^{\prime}$ decay,

$\sigma\left(p p \rightarrow W^{\prime} X \rightarrow W Z X\right)=\sigma\left(p p \rightarrow W^{\prime} X\right) \times \mathrm{BR}\left(W^{\prime} \rightarrow W Z\right)$.

Here, $\sigma\left(p p \rightarrow W^{\prime} X\right)$ is the total (theoretical) $W^{\prime}$ production cross section and $\mathrm{BR}\left(W^{\prime} \rightarrow W Z\right)=\Gamma_{W^{\prime}}^{W Z} / \Gamma_{W^{\prime}}$ with $\Gamma_{W^{\prime}}$ the total width of $W^{\prime}$. "Narrow" refers to the assumption that the natural width of a resonance is smaller than the typical experimental resolution of $5 \%$ of its mass [43,44], which is true for a large fraction of the parameter space of the reference EGM model.

Likewise, $Z^{\prime}$ production and decay into $W W$ can be observed through resonant pair production of charged gauge bosons $W W$. In the NWA, one can write down the cross section of process (1.4) as follows:

$\sigma\left(p p \rightarrow Z^{\prime} X \rightarrow W W X\right)=\sigma\left(p p \rightarrow Z^{\prime} X\right) \times \mathrm{BR}\left(Z^{\prime} \rightarrow W W\right)$.

Here, $\sigma\left(p p \rightarrow Z^{\prime} X\right)$ is the total (theoretical) $Z^{\prime}$ production cross section and $\operatorname{BR}\left(Z^{\prime} \rightarrow W W\right)=\Gamma_{Z^{\prime}}^{W W} / \Gamma_{Z^{\prime}}$ with $\Gamma_{Z^{\prime}}$ the total width of $Z^{\prime}$.

\section{$4 W^{\prime}$ and $Z^{\prime}$ Branching Ratios}

We shall here review the decay modes of $W^{\prime}$ and $Z^{\prime}$, with a focus on their branching ratios to $W Z$ and $W W$, respectively. 


\section{$4.1 W^{\prime} \rightarrow W Z$}

In the EGM the $W^{\prime}$ bosons can decay into SM fermions, gauge bosons $(W Z)$, or a pair of the charged SM $W$ boson and the Higgs boson $H$. In the calculation of the total width $\Gamma_{W^{\prime}}$ we consider the following channels: $W^{\prime} \rightarrow f \bar{f}^{\prime}, W Z$, and $W H$, where $f$ are SM fermions $(f=\ell, v, q) .{ }^{6}$ Only left-handed neutrinos are considered, while possible righthanded neutrinos are assumed to be kinematically unavailable as final states. Also, throughout the paper we shall ignore the couplings of the $W^{\prime}$ to other beyond-SM particles such as SUSY partners and exotic fermions in the theory. The presence of such channels would increase the width of the $W^{\prime}$ and hence lower the branching ratio into a $W Z$ pair. As a result, the total decay width of the $W^{\prime}$ boson is taken to be

$\Gamma_{W^{\prime}}=\sum_{f} \Gamma_{W^{\prime}}^{f \bar{f}^{\prime}}+\Gamma_{W^{\prime}}^{W Z}+\Gamma_{W^{\prime}}^{W H}$.

The fermion contribution, $\sum_{f f^{\prime}} \Gamma_{W^{\prime}}^{f \bar{f}^{\prime}}$, would depend on the number $n_{g}$ of generations of heavy exotic fermions which can contribute to the $W^{\prime}$ decay without phase space suppression. This number is model dependent too, and introduces a phenomenological uncertainty. The presence of the last two decay channels, which are often neglected at low and moderate values of $M_{W^{\prime}}$, is due to $W-W^{\prime}$ mixing which is constrained to be tiny. In particular, for the range of $M_{W^{\prime}}$ values below $\sim 1.0-1.5 \mathrm{TeV}$, the dependence of $\Gamma_{W^{\prime}}$ on the values of $\xi_{W-W^{\prime}}$ (within its allowed range) induced by $\Gamma_{W^{\prime}}^{W Z}$ and $\Gamma_{W^{\prime}}^{W H}$ is unimportant because $\sum_{f} \Gamma_{W^{\prime}}^{f \bar{f}^{\prime}}$ dominates over diboson partial widths. Therefore, in this mass range, one can approximate the total width as $\Gamma_{W^{\prime}} \approx \sum_{f} \Gamma_{W^{\prime}}^{f \bar{f}^{\prime}}=$ $3.5 \% \times M_{W^{\prime}}$ [40], where the sum runs over SM fermions only.

For heavier $W^{\prime}$ bosons, the diboson decay channels, $W Z$ and $W H$, start to play an important role, and we are no longer able to ignore them [40]. To be specific, we take an approach as model-independent as possible, and for numerical illustration show our results in two simple scenarios. In the first scenario, we treat the model as effectively having a negligible partial width of $W^{\prime} \rightarrow W H$ with respect to that of $W^{\prime} \rightarrow W Z$, i.e. $\Gamma_{W^{\prime}}^{W H} \ll \Gamma_{W^{\prime}}^{W Z}$, so that one can ignore the former, taking $\Gamma_{W^{\prime}}^{W H} \simeq 0$. In this case, numerical results with our treatment will serve as an upper bound on the size of the signal. The second scenario assumes that both partial widths are comparable, $\Gamma_{W^{\prime}}^{W H} \simeq \Gamma_{W^{\prime}}^{W Z}$ for heavy $M_{W^{\prime}}$, as required by the "Equivalence theorem" [45].

In the first scenario, where $\Gamma_{W^{\prime}}^{W H}=0$, for a fixed mixing factor $\xi_{W-W^{\prime}}$ and at large $M_{W^{\prime}}$, where $\Gamma_{W^{\prime}}^{W Z}$ dominates over $\sum_{f} \Gamma_{W^{\prime}}^{f \bar{f}^{\prime}}$, the total width increases rapidly with the $W^{\prime}$

\footnotetext{
${ }^{6}$ Here, the $\ell$ includes $\tau$ leptons.
}

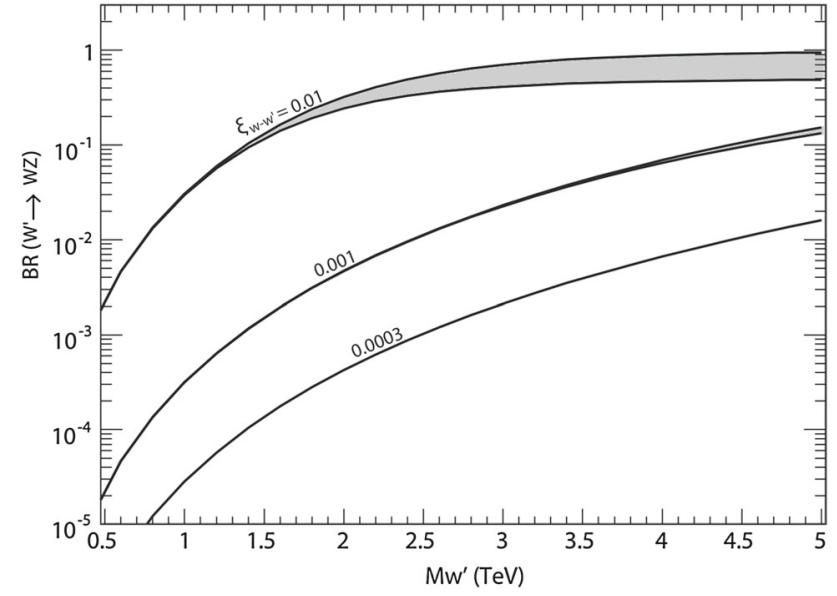

Fig. 1 Branching fraction $\mathrm{BR}\left(W^{\prime} \rightarrow W Z\right)$ (solid) vs $M_{W^{\prime}}$ in the EGM for $W-W^{\prime}$ mixing factor $\xi_{W-W^{\prime}}=3 \cdot 10^{-4}, 10^{-3}$ and $10^{-2}$. The shaded bands represent the uncertainty resulting from the inclusion of the $\mathrm{WH}$ decay mode, the upper and lower bounds correspond to the assumptions $\Gamma_{W^{\prime}}^{W H}=0$ and $\Gamma_{W^{\prime}}^{W H}=\Gamma_{W^{\prime}}^{W Z}$, respectively

Table $1 W^{\prime}$ branching ratios in per cent for $\xi_{W-W^{\prime}}=\left(M_{W} / M_{W^{\prime}}\right)^{2}$

\begin{tabular}{lrrrrr}
\hline$M_{W^{\prime}}(\mathrm{TeV})$ & \multicolumn{1}{l}{1} & \multicolumn{1}{l}{3} & \multicolumn{1}{l}{4} & \multicolumn{1}{c}{5} \\
\hline $\mathrm{BR} \rightarrow$ light quarks & 49.3 & 48.9 & 48.9 & 48.8 & 48.8 \\
$\mathrm{BR} \rightarrow t \bar{b}$ & 23.6 & 24.2 & 24.3 & 24.4 & 24.4 \\
$\mathrm{BR} \rightarrow \ell v$ & 24.7 & 24.5 & 24.4 & 24.4 & 24.4 \\
$\mathrm{BR} \rightarrow W Z+W H$ & 2.4 & 2.4 & 2.4 & 2.4 & 2.4 \\
\hline
\end{tabular}

mass because of the quintic dependence on the $M_{W^{\prime}}$ mass of the $W Z$ mode, $\Gamma_{W^{\prime}}^{W Z} \propto M_{W^{\prime}}\left[M_{W^{\prime}}^{4} /\left(M_{W}^{2} M_{Z}^{2}\right)\right]$, corresponding to the production of longitudinally polarized $W$ and $Z$ in the channel $W^{\prime} \rightarrow W_{L} Z_{L}[2,40]$. In this case, the $W Z$ mode becomes dominant and $\mathrm{BR}\left(W^{\prime} \rightarrow W Z\right) \rightarrow 1$, while the fermionic decay channels, $\sum_{f} \Gamma_{W^{\prime}}^{f \bar{f}^{\prime}} \propto M_{W^{\prime}}$, are increasingly suppressed. However, in the second scenario with $\Gamma_{W^{\prime}}^{W H}=\Gamma_{W^{\prime}}^{W Z}, \mathrm{BR}\left(W^{\prime} \rightarrow W Z\right) \rightarrow 0.5$ when $M_{W^{\prime}}$ increases, as illustrated in Fig. 1.

Before closing the discussion of the diboson branching ratios, we compare them in Tables 1 and 2 to those for fermionic final states. Two cases are considered: in Table 1, a $W^{\prime}$ with mixing $\xi_{W-W^{\prime}}=\left(M_{W} / M_{W^{\prime}}\right)^{2}$, as suggested by Eq. (2.1) with $\mathcal{C}=1$, and in Table 2 , a $W^{\prime}$ with mixing $\xi_{W-W^{\prime}}=10^{-3}$. In evaluation of the diboson decay partial widths of $W^{\prime}$ in both cases, the relation of $\Gamma_{W^{\prime}}^{W H}=\Gamma_{W^{\prime}}^{W Z}$ is assumed. The presence of the two last diboson decay channels is due to $W-W^{\prime}$ mixing and is often neglected, however for large $W^{\prime}$ masses there is an enhancement that cancels the suppression due to the mixing leading to a linear increase of the diboson partial widths with $M_{W^{\prime}}$. This is in contrast to the second case where for a fixed (mass-independent) value of $\xi_{W-W^{\prime}}$, the diboson branching ratio is seen to grow rapidly 
Table $2 W^{\prime}$ branching ratios in per cent for $\xi_{W-W^{\prime}}=10^{-3}$

\begin{tabular}{lrrrrl}
\hline$M_{W^{\prime}}(\mathrm{TeV})$ & \multicolumn{1}{l}{1} & \multicolumn{1}{l}{2} & \multicolumn{1}{l}{3} & \multicolumn{1}{l}{4} & \multicolumn{1}{l}{5} \\
\hline $\mathrm{BR} \rightarrow$ light quarks & 50.5 & 49.7 & 47.7 & 43.4 & 36.4 \\
$\mathrm{BR} \rightarrow t \bar{b}$ & 24.1 & 24.5 & 23.8 & 21.6 & 18.2 \\
$\mathrm{BR} \rightarrow \ell v$ & 25.3 & 24.8 & 23.9 & 21.7 & 18.2 \\
$\mathrm{BR} \rightarrow W Z+W H$ & 0.1 & 1.0 & 4.6 & 13.3 & 27.2 \\
\hline
\end{tabular}

with mass, in fact as the fifth power [2]. This feature of the model allows for a high sensitivity.

\section{$4.2 Z^{\prime} \rightarrow W W$}

In analogy with the $W^{\prime}$ case, in the calculation of the total width $\Gamma_{Z^{\prime}}$ we included $Z^{\prime} \rightarrow f \bar{f}, W^{+} W^{-}$, and $Z H[39,46]$. We shall again ignore the couplings of the $Z^{\prime}$ to any beyondSM particles such as right-handed neutrinos, SUSY partners or exotic fermions in the theory, which may increase the width of the $Z^{\prime}$ and hence lower the branching ratio into a pair of $W^{ \pm}$by the same factor. The total width $\Gamma_{Z^{\prime}}$ of the $Z^{\prime}$ boson can then be written as:

$\Gamma_{Z^{\prime}}=\sum_{f} \Gamma_{Z^{\prime}}^{f f}+\Gamma_{Z^{\prime}}^{W W}+\Gamma_{Z^{\prime}}^{Z H}$

Similar to the total decay width of the $W^{\prime}$ boson defined in Eq. (4.1), the presence of the two last decay channels is due to $Z-Z^{\prime}$ mixing. Note, that the widths of these two bosonic modes $W^{+} W^{-}$and $Z H$ do not depend on unknown masses of the final states. For the range of $M_{Z^{\prime}}$ values below $\sim 3 \mathrm{TeV}$, the dependence of $\Gamma_{Z^{\prime}}$ on the values of the mixing parameter $\xi_{Z-Z^{\prime}}$ [2] (within its allowed range) induced by $\Gamma_{Z^{\prime}}^{W W}$ and $\Gamma_{Z^{\prime}}^{Z H}$ is unimportant. Therefore, in this mass range, one can approximate the total width as $\Gamma_{Z^{\prime}} \approx \sum_{f} \Gamma_{Z^{\prime}}^{f f}$, where the sum runs over SM fermions only. In this mass range, the ratio of $\Gamma_{Z^{\prime}} / M_{Z^{\prime}}=0.03$ for the EGM from which one can appreciate the narrowness of the $Z^{\prime}$ pole.

However, for large $Z^{\prime}$ masses, $M_{Z^{\prime}}>3-5 \mathrm{TeV}$, there is an enhancement that cancels the suppression due to the tiny $Z-Z^{\prime}$ mixing parameter $\xi_{Z-Z^{\prime}}$ [39]. While the "Equivalence theorem" [45] might suggest a value for $\mathrm{BR}\left(Z^{\prime} \rightarrow Z H\right)$ comparable to $\mathrm{BR}\left(Z^{\prime} \rightarrow W^{+} W^{-}\right)$up to electroweak symmetry breaking effects and phase-space factors, the $Z^{\prime} Z H$ coupling is quite model dependent $[46,47]$. We again take an approach as model-independent as possible, and show our results for two scenarios, analogous to the corresponding ones for the $W^{\prime}$ case. In the first scenario, we treat the model as effectively having a suppressed partial width of $Z^{\prime} \rightarrow Z H$ with respect to that of $Z^{\prime} \rightarrow W^{+} W^{-}$, i.e. $\Gamma_{Z^{\prime}}^{Z H} \ll \Gamma_{Z^{\prime}}^{W W}$, so that one can ignore the former. In this case, numerical results with our treatment will serve as an upper bound on the size of the signal. The second scenario concerns the situation when

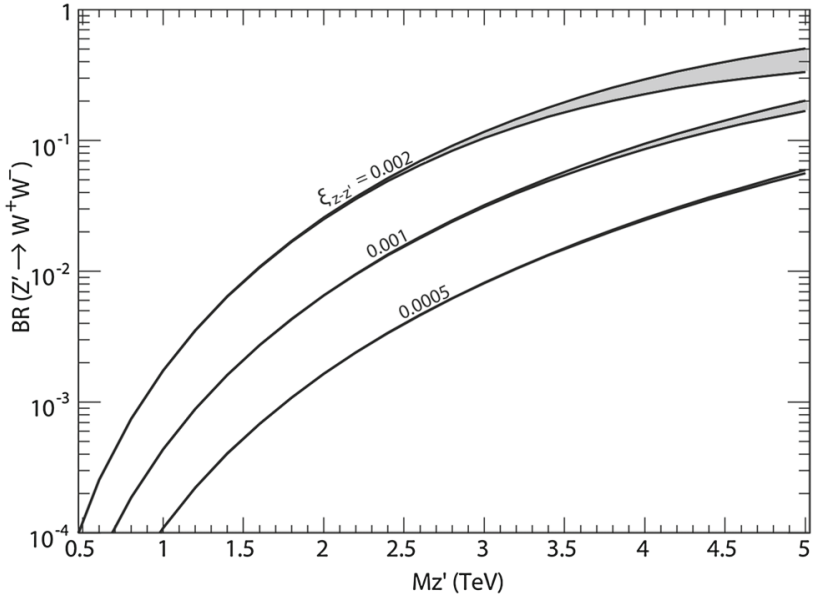

Fig. 2 Branching fraction $\operatorname{BR}\left(Z^{\prime} \rightarrow W^{+} W^{-}\right)$vs. $M_{Z^{\prime}}$ in the EGM for non-zero $Z-Z^{\prime}$ mixing factor $\xi_{Z-Z^{\prime}}=2 \cdot 10^{-3}, 1 \cdot 10^{-3}$ and $5 \cdot 10^{-4}$. The shaded bands represent the uncertainty resulting from the inclusion of the $Z H$ decay mode to the total decay width $\Gamma_{Z^{\prime}}$, the upper and lower bounds correspond to the assumptions $\Gamma_{Z^{\prime}}^{Z H}=0$ and $\Gamma_{Z^{\prime}}^{Z H}=\Gamma_{Z^{\prime}}^{W^{+} W^{-}}$, respectively

both partial widths are comparable, $\Gamma_{Z^{\prime}}^{Z H} \simeq \Gamma_{Z^{\prime}}^{W W}$ for heavy $M_{Z^{\prime}}$ [46-48].

For a fixed mixing factor $\xi_{Z-Z^{\prime}}$ and at large $M_{Z^{\prime}}$ where $\Gamma_{Z^{\prime}}^{W W}$ dominates over $\sum_{f} \Gamma_{Z^{\prime}}^{f f}$ (assuming partial width of $\Gamma_{Z^{\prime}}^{Z H}=0$ ) the total width increases rapidly with the mass $M_{Z^{\prime}}$ because of the quintic dependence on the $Z^{\prime}$ mass of the $W^{+} W^{-}$mode $[2,39]$. In this case, the $W^{+} W^{-}$mode becomes dominant and $\operatorname{BR}\left(Z^{\prime} \rightarrow W^{+} W^{-}\right) \rightarrow 1$, while the fermionic decay channels $\left(\Gamma_{Z^{\prime}}^{f f} \propto M_{Z^{\prime}}\right)$ are increasingly subdominant.

For the EGM, the $Z-Z^{\prime}$ mixing parameter $\xi_{Z-Z^{\prime}}$ is constrained at the level of a few per mil [17] from an analysis of the $Z^{\prime}$ model against available electroweak precision data, resulting in $\xi_{Z-Z^{\prime}}^{\mathrm{EW}}<2.6 \cdot 10^{-3}$. In Fig. 2 we plot $\mathrm{BR}\left(Z^{\prime} \rightarrow W^{+} W^{-}\right)$vs $M_{Z^{\prime}}$ for the EGM and mixing factor $\xi_{Z-Z^{\prime}}$ ranging from 0.0005 to 0.002 . The case when $\Gamma_{Z^{\prime}}^{Z H}=\Gamma_{Z^{\prime}}^{W^{+} W^{-}}$is also shown in Fig. 2.

It should be stressed that the boost of the branching ratio for high values of $M_{W^{\prime}}$ and $M_{Z^{\prime}}$, illustrated in Figs. 1 and 2, plays an important role in the following analysis.

\section{Analysis}

\subsection{Production and decay of $W^{\prime} \rightarrow W Z$}

Here, we present an analysis, employing the most recent measurements of diboson processes provided by ATLAS [37] with the full Run 2 data set with time-integrated luminosity of $139 \mathrm{fb}^{-1}$ as well as, for the sake of comparison, with a partial Run 2 data set with time integrated luminosity of $36.7 \mathrm{fb}^{-1}$ 


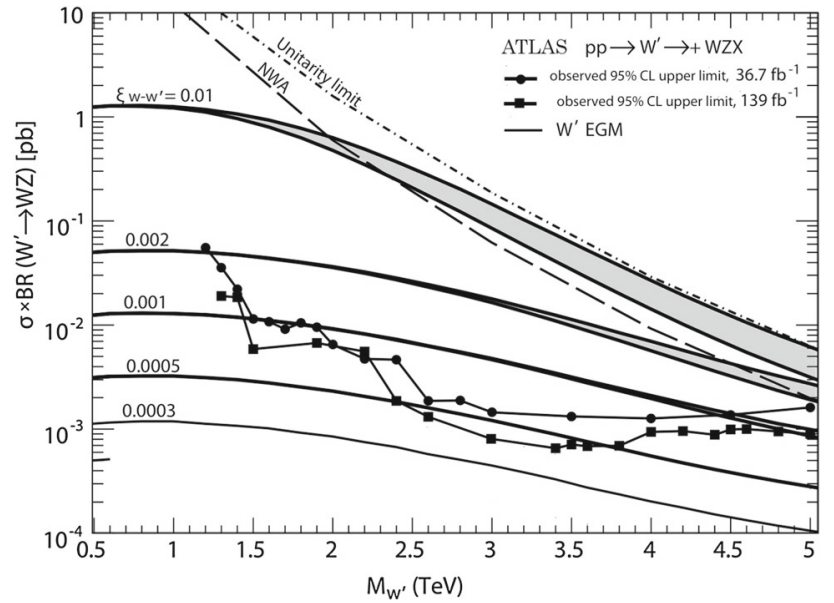

Fig. 3 Observed 95\% CL upper limits on the production cross section times the branching fraction, $\sigma_{95} \% \times \mathrm{BR}\left(W^{\prime} \rightarrow W Z\right)$, as a function of the $W^{\prime}$ mass, $M_{W^{\prime}}$, showing ATLAS data on the fully hadronic final states for $36.7 \mathrm{fb}^{-1}$ [36] and $139 \mathrm{fb}^{-1}$ [37]. The theoretical production cross sections $\sigma\left(p p \rightarrow W^{\prime}+X\right) \times \mathrm{BR}\left(W^{\prime} \rightarrow W Z\right)$ for the EGM are calculated from PYTHIA with a $W^{\prime}$ boson mass-dependent $K$-factor used to correct for NNLO QCD effects, and given by solid curves, for mixing factor $\xi_{W-W^{\prime}}$ ranging from $10^{-2}$ and down to $3 \cdot 10^{-4}$. The shaded bands are defined like in Fig. 1. The area lying below the long-dashed curve labelled NWA corresponds to the region where the narrow-resonance assumption is satisfied. The lower boundary of the region excluded by the unitarity violation arguments is indicated by the dot-dashed curve $[40,49]$

[36]. As mentioned above, ATLAS analyzed the $W Z$ production in the process (1.3) through the fully hadronic $(q q q q)$ final states. ${ }^{7}$ In Fig. 3, we show the observed 95\% CL upper limits on the production cross section times the branching fraction, $\sigma_{95} \% \times \mathrm{BR}\left(W^{\prime} \rightarrow W Z\right)$, as a function of the $W^{\prime}$ mass, $M_{W^{\prime}}$. The data analyzed comprises $p p$ collisions at $\sqrt{s}=13 \mathrm{TeV}$, recorded by the ATLAS $\left(36.7 \mathrm{fb}^{-1}\right.$ and $139 \mathrm{fb}^{-1}$ ) detector $[36,37]$ at the LHC.

Then, for $W^{\prime}$ we compute the LHC theoretical production cross section multiplied by the branching ratio into $W Z$ bosons, $\sigma\left(p p \rightarrow W^{\prime} X\right) \times \mathrm{BR}\left(W^{\prime} \rightarrow W Z\right)$, as a function of the two parameters $\left(M_{W^{\prime}}, \xi_{W-W^{\prime}}\right)$ [40], and compare it with the limits established by the ATLAS experiment, $\sigma_{95 \%} \times \mathrm{BR}\left(W^{\prime} \rightarrow W Z\right)$. The simulation of signals for the EGM $W^{\prime}$ is based on an adapted version of the leading order (LO) PYHTHIA 8.2 event generator [50]. A mass-dependent $K$ factor is adopted to rescale the LO PYTHIA prediction to the next-to-next-to-leading-order (NNLO) in $\alpha_{s}$. The theoretical $W^{\prime}$ production cross section $\sigma\left(p p \rightarrow W^{\prime} X\right)$ is scaled to an NNLO calculation in $\alpha_{s}$ by ZWPROD [51], given by solid curves, and shown in Fig. 3 for a mixing factor $\xi_{W-W^{\prime}}$ ranging from $10^{-2}$ and down to $3 \cdot 10^{-4}$. The factorization and renormalization scales are set to the $W^{\prime}$ resonance mass.

\footnotetext{
${ }^{7}$ For the experimental data, " $q q q q$ " refers to four-jet final states (including gluons).
}

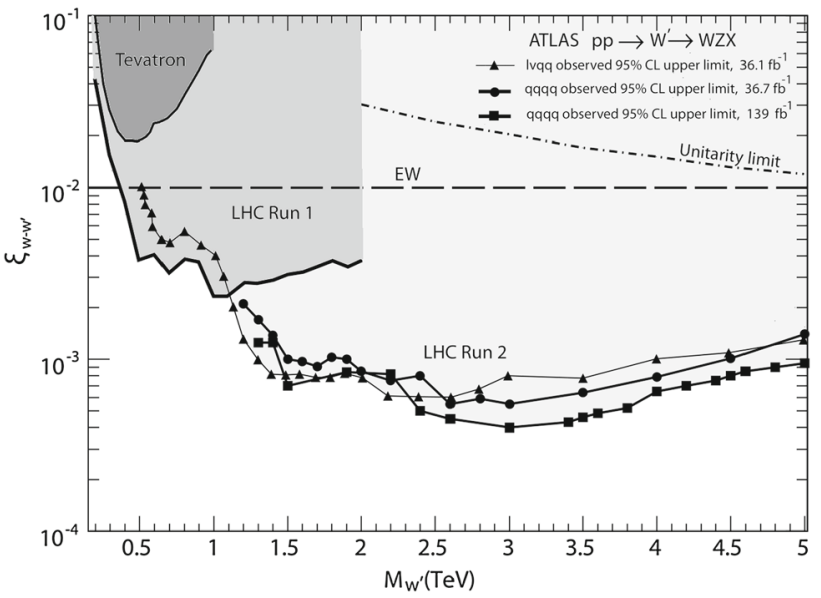

Fig. $495 \% \mathrm{CL}$ exclusion regions in the two-dimensional $\left(M_{W^{\prime}}\right.$, $\left.\xi_{W-W^{\prime}}\right)$ plane obtained from the precision electroweak data (horizontal dashed straight line labeled "EW"), the direct search constraints at the Tevatron in $p \bar{p} \rightarrow W Z X$ (the dark shaded area) as well as from the LHC measurement of $p p \rightarrow W Z X$ at $7 \mathrm{TeV}$ and $8 \mathrm{TeV}$ (Run 1) (the gray area) and at $13 \mathrm{TeV}$ from diboson $W^{\prime} \rightarrow W Z$ production in hadronic final states using the partial and full Run 2 ATLAS data set. Limits obtained from the semileptonic channel $\ell v q q$ at time-integrated luminosity of $36.1 \mathrm{fb}^{-1}$ [40] are overlaid for comparison. The combined exclusion region for the EGM $W^{\prime}$ boson obtained after incorporating direct search constraints from the LHC Run 2 data set is shown as the light shaded area. The uninarity limit is shown as a dot-dashed curve

As was explained in connection with Fig. 1, the upper (lower) boundary of the shaded areas correspond to a scenario where the contribution of the decay channel $W^{\prime} \rightarrow W H$ to the total $W^{\prime}$ decay width of Eq. (4.1) is $\Gamma_{W^{\prime}}^{W H}=0$ $\left(\Gamma_{W^{\prime}}^{W H}=\Gamma_{W^{\prime}}^{W Z}\right)$. The area below the long-dashed curve labelled "NWA" corresponds to the region where the $W^{\prime}$ resonance width is predicted to be less than $5 \%$ of its mass, corresponding to the best detector resolution of the searches, where the narrow-resonance assumption is satisfied. We also show a curve labelled "Unitarity limit" that corresponds to the unitarity bound (see, e.g. [49] and references therein). In that paper, it was shown that the saturation of unitarity in the elastic scattering $W^{ \pm} Z \rightarrow W^{ \pm} Z$ leads to the constraint $g_{W^{\prime} W Z \max }=g_{W W Z} \cdot M_{Z}^{2} /\left(\sqrt{3} M_{W^{\prime}} M_{W}\right)$ that was exploited in plotting the unitarity bound. This constraint was obtained under the assumption that the couplings of the $W^{\prime}$ to quarks and to gauge bosons have the same Lorentz structure as those of the SM but with rescaled strength.

The theoretical curves for the cross sections $\sigma(p p \rightarrow$ $\left.W^{\prime} X\right) \times \mathrm{BR}\left(W^{\prime} \rightarrow W Z\right)$, in descending order, correspond to values of the $W-W^{\prime}$ mixing factor $\xi_{W-W^{\prime}}$ from 0.01 to 0.0003 . The intersection points of the measured upper limits on the production cross section with these theoretical cross sections for various values of $\xi_{W-W^{\prime}}$ give the corresponding lower bounds on $\left(M_{W^{\prime}}, \xi_{W-W^{\prime}}\right)$, displayed in Fig. 4.

The limits arising from the diboson channel are basically excluding large values of $\xi_{W-W^{\prime}}$, strongest at intermediate 
masses $M_{W^{\prime}} \sim 2-4 \mathrm{TeV}$, as illustrated in Fig. 4. Interestingly, Fig. 4 shows that at moderate and high $W^{\prime}$ masses, the limits on $\xi_{W-W^{\prime}}$ obtained from the ATLAS diboson resonance production search at $13 \mathrm{TeV}$ and at time-integrated luminosity of $139 \mathrm{fb}^{-1}$ are substantially stronger than those derived from the low-energy electroweak data, which are of the order $\sim 10^{-2}[1]$, as well as those obtained from the partial ATLAS Run 2 data set with time integrated luminosity of $36.7 \mathrm{fb}^{-1}$ [36] in the fully hadronic final states, and as well as those obtained in the semileptonic final state at $36.1 \mathrm{fb}^{-1}$ [40].

Comparison of sensitivities to $W^{\prime}$ of the process (1.3) with different decay channels, e.g., $V V \rightarrow \ell v q q$ and $q q q q$, can be performed by the matching of $95 \%$ CL upper limits on the production cross section times the branching fraction, $\sigma_{95 \%} \times \mathrm{BR}\left(W^{\prime} \rightarrow W Z\right)$, which includes the SM branching fractions of the electroweak bosons to the final states in the analysis channel, effects from detector acceptance, as well as reconstruction and selection efficiencies. ATLAS bounds were included according to HEPdata [52]. From a comparison of the upper limits on the production cross section times the branching fraction for semileptonic $\ell v q q$ vs. fully hadronic $q q q q$ decay channels at $36.1 \mathrm{fb}^{-1}$ and $36.7 \mathrm{fb}^{-1}$, respectively, one can conclude that the $q 9 q q$ channel dominates the sensitivity in the higher resonance mass range $\left(2.6 \mathrm{TeV} \leq M_{W^{\prime}} \leq 5 \mathrm{TeV}\right)$, while at lower masses the sensitivity of the semileptonic channel dominates over the fully hadronic one. These features are illustrated in Fig. 4.

For reference, we display limits on the $W^{\prime}$ parameters from the Tevatron (CDF and D0) as well as from ATLAS and CMS obtained at 7 and $8 \mathrm{TeV}$ of the LHC data taking in Run 1 denoted as "LHC Run 1" [40]. Figure 4 shows that the experiments CDF and D0 at the Tevatron exclude EGM $W^{\prime}$ bosons with $\xi_{W-W^{\prime}} \gtrsim 2 \cdot 10^{-2}$ in the resonance mass range $0.25 \mathrm{TeV}<M_{W^{\prime}}<1 \mathrm{TeV}$ at the $95 \% \mathrm{CL}$, whereas LHC in Run 1 improved those constraints, excluding $W^{\prime}$ boson parameters at $\xi_{W-W^{\prime}} \gtrsim 2 \cdot 10^{-3}$ in the mass range $0.2 \mathrm{TeV}<M_{W^{\prime}}<2 \mathrm{TeV}$.

As expected, the increase of the time-integrated luminosity up to $139 \mathrm{fb}^{-1}$ leads to dominant sensitivity of the $q q q q$ channel over the whole resonance mass range of $1.3 \mathrm{TeV}<$ $M_{W^{\prime}}<5 \mathrm{TeV}$ and it allows to set stronger constraints on the mixing angle $\xi_{W-W^{\prime}}$, resulting in $\xi_{W-W^{\prime}}>4.3 \cdot 10^{-4}$ as shown in Fig. 4. Our results extend the sensitivity beyond the corresponding CDF Tevatron results [35] as well as the ATLAS and CMS sensitivity attained at 7 and $8 \mathrm{TeV}$. Also, for the first time, we set $W^{\prime}$ limits as functions of the mass $M_{W^{\prime}}$ and mixing factor $\xi_{W-W^{\prime}}$ at the LHC at $13 \mathrm{TeV}$ with the partial ATLAS Run 2 data set at time-integrated luminosity of $36.7 \mathrm{fb}^{-1}$ [36], and with the full ATLAS Run 2 data set with a time-integrated luminosity of $139 \mathrm{fb}^{-1}$. The exclusion region obtained in this way on the parameter space of the $W^{\prime}$ from the full Run 2 data set supersedes the corresponding exclusion area obtained at the LHC at $\sqrt{s}=13 \mathrm{TeV}$ and time-integrated luminosity of $36.1 \mathrm{fb}^{-1}$ in the semileptonic channel as reported in [40]. The limits on $W^{\prime}$ parameters presented in this section obtained from the diboson $W Z$ production in hadronic final states using the full Run 2 ATLAS data set, corresponding to a time-integrated luminosity of $139 \mathrm{fb}^{-1}$ are the best to date.

\subsection{Production and decay of $Z^{\prime} \rightarrow W W$}

For the $Z^{\prime}$ case, the analysis proceeds in a similar fashion. We show in Fig. 5 the observed 95\% CL upper limits on the production cross section times the branching fraction, $\sigma_{95 \%} \times \operatorname{BR}\left(Z^{\prime} \rightarrow W^{+} W^{-}\right)$, as a function of the $Z^{\prime}$ mass, $M_{Z^{\prime}}$. Then, for $Z^{\prime}$ we compute the LHC production cross section multiplied by the branching ratio into two $W$ bosons, $\sigma \times \operatorname{BR}\left(Z^{\prime} \rightarrow W^{+} W^{-}\right)_{\text {theory }}$, as a function of the two parameters $\left(M_{Z^{\prime}}, \xi_{Z-Z^{\prime}}\right)$, and compare it with the limits established by the ATLAS experiment, $\sigma_{95} \% \times \mathrm{BR}\left(Z^{\prime} \rightarrow W^{+} W^{-}\right)$. Our strategy in the present analysis is to adopt the SM backgrounds that have been carefully evaluated by the experimental collaborations and contained in $\sigma_{95} \% \times \mathrm{BR}\left(Z^{\prime} \rightarrow\right.$ $W^{+} W^{-}$) and simulate only the $Z^{\prime}$ signal. Comparison of the 95\% CL upper limits on the production cross section times the branching fraction, $\sigma_{95 \%} \times \mathrm{BR}\left(Z^{\prime} \rightarrow W^{+} W^{-}\right)$, as a function of the $Z^{\prime}$ mass based on the ATLAS data of the fully hadronic final states for $36.7 \mathrm{fb}^{-1}$ [36] and $139 \mathrm{fb}^{-1}$ [37] demonstrates the dominating sensitivity to $Z^{\prime}$ of the latter time-integrated luminosity data with respect to the former one, over the whole $Z^{\prime}$ mass range.

In Fig. 5, the theoretical production cross section $\sigma \times$ $\mathrm{BR}\left(Z^{\prime} \rightarrow W^{+} W^{-}\right)$theory for $Z^{\prime}$ boson of the EGM, is calculated from PYTHIA 8.2 [50] adapted for such kind of analysis. Higher-order QCD corrections to the signal were estimated using a $K$-factor, for which we adopt a massindependent value of 1.9 [53-55]. These theoretical curves for the cross sections, in descending order, correspond to values of the $Z-Z^{\prime}$ mixing factor $\xi_{Z-Z^{\prime}}$ from 0.002 to 0.0003 . The intersection points of the expected measured upper limits on the production cross section with this theoretical cross section for various values of $\xi_{Z-Z^{\prime}}$ give the corresponding lower bounds on $\left(M_{Z^{\prime}}, \xi_{Z-Z^{\prime}}\right)$, to be presented in Fig. 6. For reference, we plot also a curve labelled "Unitarity limit" that corresponds to the unitarity bound [39,49]. In [49], it was shown that the saturation of unitarity in the elastic scattering $W^{+} W^{-} \rightarrow W^{+} W^{-}$leads to the constraint $g_{Z^{\prime} W W_{\max }}=g_{Z W W} \cdot\left(M_{Z} / \sqrt{3} M_{Z^{\prime}}\right)$ that was exploited in plotting the unitarity bound.

Different bounds on the $Z^{\prime}$ parameter space are collected in Fig. 6, showing that at high $Z^{\prime}$ masses, the limits on $\xi_{Z-Z^{\prime}}$ obtained from the full Run 2 data set collected at $\sqrt{s}=13$ $\mathrm{TeV}$ and recorded by the ATLAS detector are substantially stronger than those derived from the global analysis of the 


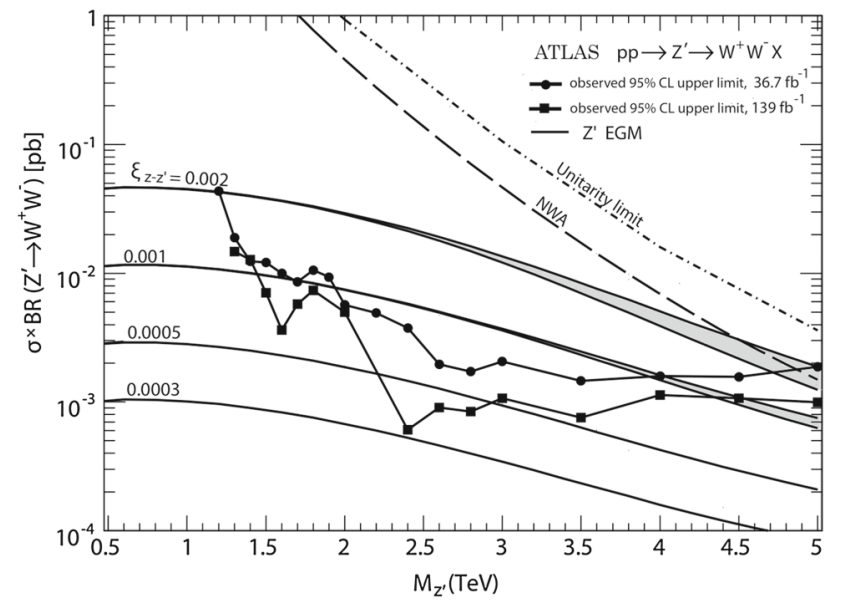

Fig. 5 Observed 95\% CL upper limits on the production cross section times the branching fraction, $\sigma_{95 \%} \times \mathrm{BR}\left(Z^{\prime} \rightarrow W^{+} W^{-}\right)$, as a function of the $Z^{\prime}$ mass, $M_{Z^{\prime}}$, showing ATLAS data of the fully hadronic final states for $36.7 \mathrm{fb}^{-1}$ [36] and $139 \mathrm{fb}^{-1}$ [37]. Theoretical production cross sections $\sigma\left(p p \rightarrow Z^{\prime}+X\right) \times \mathrm{BR}\left(Z^{\prime} \rightarrow W^{+} W^{-}\right)$for the EGM are calculated from PYTHIA with a $K$-factor used to correct for NNLO QCD effects, and given by solid curves, for mixing factors $\xi_{Z-Z^{\prime}}$ ranging from $2 \cdot 10^{-3}$ and down to $3 \cdot 10^{-4}$. The shaded bands are defined like in Fig. 2. The area lying below the long-dashed curve labelled NWA corresponds to the region where the narrow-resonance assumption is satisfied. The lower boundary of the region excluded by the unitarity violation arguments is also indicated by the dot-dashed curve [39,49]

precision electroweak data [17], which is also displayed. In this Fig. 6, we display limits on the $Z^{\prime}$ parameters in the EGM from the Tevatron exclusion [35], as well as those derived from the CMS measurement of $p p \rightarrow W W X$ in Run 1 [56].

Limits obtained from the semileptonic channel $\ell v q q$ are also shown for comparison [39]. Below (above) a resonance mass value of about $2.2 \mathrm{TeV}(3 \mathrm{TeV}$ ), the semileptonic channel at time-integrated luminosity of $36.1 \mathrm{fb}^{-1}$ dominates the sensitivity, while in the resonance mass range $2.2 \mathrm{TeV} \leq M_{Z^{\prime}} \leq 3 \mathrm{TeV}$ the all-hadronic channel at luminosity of $139 \mathrm{fb}^{-1}$ is most sensitive. As for a comparison of the sensitivities of different channels, semileptonic vs. fully hadronic final states, at the $\mathrm{LHC}$ at $13 \mathrm{TeV}$ with partial ATLAS Run 2 data set, Fig. 6 shows that the $\ell v q q$ channel dominates the sensitivity over the whole resonance mass range $0.5 \mathrm{TeV} \leq M_{Z^{\prime}} \leq 5 \mathrm{TeV}$.

In Table 3, we collect our limits on the $W^{\prime}$ and $Z^{\prime}$ parameters for the benchmark EGM model. Also shown in Table 3 are the current limits on the $W-W^{\prime}$ and $Z-Z^{\prime}$ mixing parameters, $\xi_{W-W^{\prime}}$ and $\xi_{Z-Z^{\prime}}$, from the Tevatron, derived from studies of diboson $W Z$ and $W W$ pair production. The limits on $\xi_{V-V^{\prime}}$ at the Tevatron assume (as does the present study) that no decay channels into exotic fermions or superpartners are open to the $W^{\prime}$ and $Z^{\prime}$. Otherwise, the limits would be moderately weaker. Table 3 shows that the limits on $\xi_{V-V^{\prime}}$ from the EW precision data are generally stronger than those

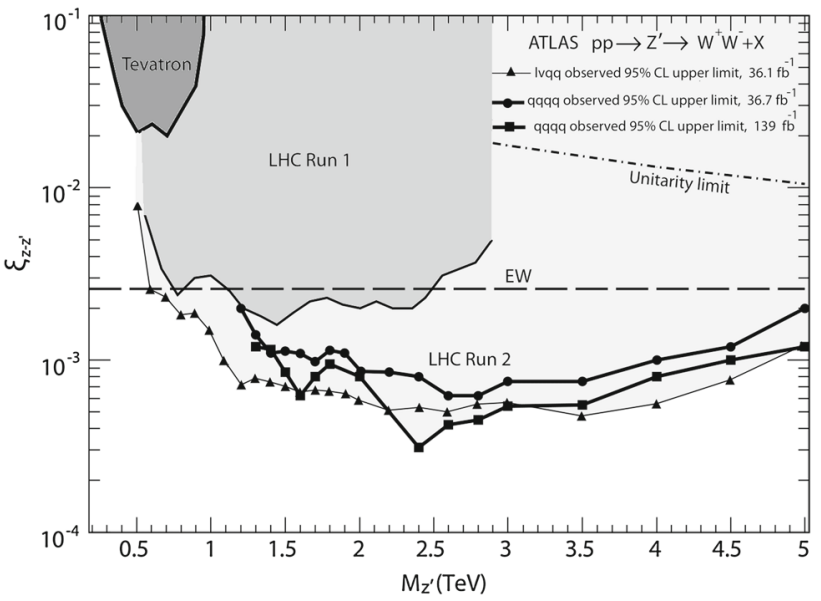

Fig. $695 \%$ CL exclusion regions in the two-dimensional $\left(M_{Z^{\prime}}, \xi_{Z-Z^{\prime}}\right)$ plane obtained after incorporating direct search constraints from the $\mathrm{CDF}$ and D0 collaborations which are referred to as Tevatron (the dark shaded area) in $p \bar{p} \rightarrow W^{+} W^{-} X$ as well as those derived from the LHC measurement of $p p \rightarrow W W X$ in Run 1 (the gray area) [56] and $13 \mathrm{TeV}$ from diboson $Z^{\prime} \rightarrow W W$ production in hadronic final states using the partial and full Run 2 ATLAS data set. Also shown is the exclusion from the precision electroweak (EW) data [17]. Limits obtained from the semileptonic channel $\ell v q q$ at time-integrated luminosity of $36.1 \mathrm{fb}^{-1}$ [39] are overlaid for comparison. Combined exclusion region for the EGM $Z^{\prime}$ boson obtained after incorporating direct search constraints from the LHC Run 2 data set is shown as the light shaded area. The uninarity limit is shown as a dot-dashed curve

from the preceding Tevatron collider. The LHC operating in Run 1 has almost the same sensitivity to mixing parameters as that reached from analysis of low-energy electroweak data. The only difference is that the Run 1 limits (as analyzed here) depend on the resonance mass $\left(M_{V^{\prime}}\right)$ whereas the EW constraints are completely independent. In addition, the LHC limits obtained in Run 2 at $13 \mathrm{TeV}$, and time-integrated luminosity, $\mathcal{L}_{\text {int }}=139 \mathrm{fb}^{-1}$, improve the EW limits by a factor of approximately one order, depending on the resonance mass.

\section{Concluding remarks}

Exploration of the diboson $W Z$ and $W W$ production at the LHC with $13 \mathrm{TeV}$ data set allows to place stringent constraints on the $W-W^{\prime}$ and $Z-Z^{\prime}$ mixing parameters as well as on the $W^{\prime}$ and $Z^{\prime}$ masses, respectively. We derived such limits by using the full ATLAS Run 2 data set recorded at the CERN LHC, with integrated luminosity of $139 \mathrm{fb}^{-1}$.

By comparing the experimental limits to the theoretical predictions for the total cross section of the $W^{\prime}$ and $Z^{\prime}$ resonant production and its subsequent decay into $W Z$ or $W W$ pairs, we show that the derived constraints on the mixing parameters, $\xi_{W-W^{\prime}}$ and $\xi_{Z-Z^{\prime}}$, for the EGM model, are substantially improved with respect to those obtained from the 
Table 3 Upper limits on mixing parameters $\xi_{W-W^{\prime}}$ and $\xi_{Z-Z^{\prime}}$ at $95 \%$ CL in the EGM, processes and experiments

\begin{tabular}{lll}
\hline Collider, process & $\xi_{W-W^{\prime}}$ & $\xi_{Z-Z^{\prime}}$ \\
\hline Tevatron, $p \bar{p} \rightarrow W^{\prime} / Z^{\prime} \rightarrow W Z / W W(\rightarrow l v q q)[35]$ & $2 \cdot 10^{-2}$ & $2 \cdot 10^{-2}$ \\
electroweak (EW) data $[1,17]$ & $\sim 10^{-2}$ & $2.6 \cdot 10^{-3}$ \\
LHC@ $13 \mathrm{TeV}$, Run 2 & & $0.4-0.9$ \\
$p p \rightarrow W^{\prime} / Z^{\prime} \rightarrow W Z / W W(\rightarrow l v q q), \mathcal{L}_{\text {int }}=36.1 \mathrm{fb}^{-1}[39,40]$ & $6.0 \cdot 10^{-4}$ & $4.7 \cdot 10^{-4}$ \\
$p p \rightarrow W^{\prime} / Z^{\prime} \rightarrow W Z / W W(\rightarrow q q q q), \mathcal{L}_{\text {int }}=36.7 \mathrm{fb}^{-1}$ (this work) & $5.5 \cdot 10^{-4}$ & $6.2 \cdot 10^{-4}$ \\
$p p \rightarrow W^{\prime} / Z^{\prime} \rightarrow W Z / W W(\rightarrow q q q q), \mathcal{L}_{\text {int }}=139 \mathrm{fb}^{-1}$ (this work) & $4.3 \cdot 10^{-4}$ & $3.1 \cdot 10^{-4}$ \\
\hline
\end{tabular}

global analysis of low energy electroweak data, as well as from the diboson production study performed at the Tevatron and those based on the LHC Run 1.

In addition, our work shows that accounting for the contribution of the $V^{\prime}$ boson decay channels, $V^{\prime} \rightarrow V H$ (where $V=W / Z$ and $\left.V^{\prime}=W^{\prime} / Z^{\prime}\right)$, to the total width $\Gamma_{V^{\prime}}$ does not dramatically affect the bounds on the mixing parameter $\xi_{V-V^{\prime}}$ obtained in the scenario of a vanishing $V H$ mode, $\Gamma_{V^{\prime}}^{V H}=0$. Namely, it turns out that for the higher resonance $V^{\prime}$ masses of our interest the constraints on $V-V^{\prime}$ mixing are relaxed very little as illustrated in Figs. 3 and 5 and discussed in Refs. [39,40].

We limited ourselves here to an analysis of the full Run 2 ATLAS data set, the corresponding CMS data set is currently unavailable.

Acknowledgements This research has been partially supported by the Abdus Salam ICTP (TRIL Programme). The work of PO has been supported by the Research Council of Norway. AAP thanks the CERN $\mathrm{TH}$ department for support.

Data Availability Statement This manuscript has no associated data or the data will not be deposited. [Authors' comment: Only public, referenced data is used.]

Open Access This article is licensed under a Creative Commons Attribution 4.0 International License, which permits use, sharing, adaptation, distribution and reproduction in any medium or format, as long as you give appropriate credit to the original author(s) and the source, provide a link to the Creative Commons licence, and indicate if changes were made. The images or other third party material in this article are included in the article's Creative Commons licence, unless indicated otherwise in a credit line to the material. If material is not included in the article's Creative Commons licence and your intended use is not permitted by statutory regulation or exceeds the permitted use, you will need to obtain permission directly from the copyright holder. To view a copy of this licence, visit http://creativecomm ons.org/licenses/by/4.0/.

Funded by SCOAP ${ }^{3}$.

\section{References}

1. M. Tanabashi et al. [Particle Data Group], Review of Particle Physics. Phys. Rev. D 98(3), 030001 (2018). https://doi.org/10. 1103/PhysRevD.98.030001
2. G. Altarelli, B. Mele, M. Ruiz-Altaba, Searching for New Heavy Vector Bosons in $p \bar{p}$ Colliders. Z. Phys. C 45, 109 (1989). https://doi.org/10.1007/BF01552335, https://doi.org/10. 1007/BF01556677 (Erratum: [Z. Phys. C 47, 676 (1990)])

3. G. Aad et al. [ATLAS Collaboration], Search for high-mass dilepton resonances using $139 \mathrm{fb}^{-1}$ of $p p$ collision data collected at $\sqrt{s}=13 \mathrm{TeV}$ with the ATLAS detector. Phys. Lett. B 796, 68 (2019). https://doi.org/10.1016/j.physletb.2019.07.016. arXiv:1903.06248 [hep-ex]

4. CMS Collaboration [CMS Collaboration], Search for a narrow resonance in high-mass dilepton final states in proton-proton collisions using $140 \mathrm{fb}^{-1}$ of data at $\sqrt{s}=13 \mathrm{TeV}$, CMS-PAS-EXO19-019

5. A. Zucchetta [ATLAS and CMS Collaborations], Heavy resonances (W', Z', jets) in ATLAS and CMS in Run 2. arXiv:1905.06607 [hep-ex]

6. CMS Collaboration [CMS Collaboration], Search for high mass resonances in dielectron final state, CMS-PAS-EXO-18-006

7. G. Aad et al. [ATLAS Collaboration], Search for a heavy charged boson in events with a charged lepton and missing transverse momentum from $p p$ collisions at $\sqrt{s}=13 \mathrm{TeV}$ with the ATLAS detector. Phys. Rev. D 100, 052013 (2019). https://doi.org/10.1103/ PhysRevD.100.052013. arXiv:1906.05609 [hep-ex]

8. P. Langacker, R.W. Robinett, J.L. Rosner, New heavy gauge bosons in $p$ p and p anti-p collisions. Phys. Rev. D 30, 1470 (1984). https:// doi.org/10.1103/PhysRevD.30.1470

9. P. Langacker, Mx Luo, Constraints on additional $Z$ bosons. Phys. Rev. D 45, 278 (1992). https://doi.org/10.1103/PhysRevD.45.278

10. P. Langacker, The physics of heavy $Z^{\prime}$ Gauge bosons. Rev. Mod. Phys. 81, 1199 (2009). https://doi.org/10.1103/RevModPhys.81. 1199. arXiv:0801.1345 [hep-ph]

11. M. Schmaltz, C. Spethmann, Two simple W' models for the early LHC. JHEP 1107, 046 (2011). https://doi.org/10.1007/ JHEP07(2011)046. arXiv:1011.5918 [hep-ph]

12. C. Grojean, E. Salvioni, R. Torre, A weakly constrained W' at the early LHC. JHEP 1107, 002 (2011). https://doi.org/10.1007/ JHEP07(2011)002. arXiv:1103.2761 [hep-ph]

13. T. Jezo, M. Klasen, I. Schienbein, LHC phenomenology of general $\mathrm{SU}(2) \mathrm{xSU}(2) \mathrm{xU}(1)$ models. Phys. Rev. D 86, 035005 (2012). https://doi.org/10.1103/PhysRevD.86.035005. arXiv:1203.5314 [hep-ph]

14. Q.H. Cao, Z. Li, J.H. Yu, C.P. Yuan, Discovery and identification of $W^{\prime}$ and $Z^{\prime}$ in SU(2) x SU(2) x U(1) models at the LHC. Phys. Rev. D 86, 095010 (2012). https://doi.org/10.1103/PhysRevD.86. 095010. arXiv:1205.3769 [hep-ph]

15. B.A. Dobrescu, Z. Liu, Heavy Higgs bosons and the $2 \mathrm{TeV}$ $W^{\prime}$ boson. JHEP 1510, 118 (2015). https://doi.org/10.1007/ JHEP10(2015)118. arXiv:1507.01923 [hep-ph]

16. H. Diaz Chavez, V. Pleitez, O.P. Ravinez, Dirac neutrinos in a $S U$ (2) left-right symmetric model. arXiv:1908.02828 [hep-ph] 
17. J. Erler, P. Langacker, S. Munir, E. Rojas, Improved constraints on Z-prime bosons from electroweak precision data. JHEP 0908, 017 (2009). https://doi.org/10.1088/1126-6708/2009/ 08/017. arXiv:0906.2435 [hep-ph]

18. J.L. Hewett, T.G. Rizzo, Low-energy phenomenology of superstring inspired E(6) models. Phys. Rep. 183, 193 (1989)

19. A. Leike, The phenomenology of extra neutral gauge bosons. Phys. Rep. 317, 143-250 (1999). [hep-ph/9805494]

20. M. Dittmar, A.S. Nicollerat, A. Djouadi, Z-prime studies at the LHC: an update. Phys. Lett. B 583, 111 (2004). https://doi.org/10. 1016/j.physletb.2003.09.103. arXiv:hep-ph/0307020

21. P. Osland, A.A. Pankov, A.V. Tsytrinov, N. Paver, Spin and model identification of $Z$ ' bosons at the LHC. Phys. Rev. D 79, 115021 (2009). https://doi.org/10.1103/PhysRevD.79.115021. arXiv:0904.4857 [hep-ph]

22. S. Godfrey, T. Martin, Z' Discovery reach at future hadron colliders: a snowmass white paper. arXiv:1309.1688 [hep-ph]

23. V.V. Andreev, P. Osland, A.A. Pankov, Precise determination of $Z-Z^{\prime}$ mixing at the CERN LHC. Phys. Rev. D 90(5), 055025 (2014). https://doi.org/10.1103/PhysRevD.90. 055025. arXiv: 1406.6776 [hep-ph]

24. A.V. Gulov, A.A. Pankov, A.O. Pevzner, V.V. Skalozub, Modelindependent constraints on the Abelian $Z^{\prime}$ couplings within the ATLAS data on the dilepton production processes at $\sqrt{s}=$ $13 \mathrm{TeV}$. Nonlin. Phenom. Complex Syst. 21(1), 21 (2018). arXiv:1803.07532 [hep-ph]

25. T. Bandyopadhyay, G. Bhattacharyya, D. Das, A. Raychaudhuri, Reappraisal of constraints on $Z^{\prime}$ models from unitarity and direct searches at the LHC. Phys. Rev. D 98(3), 035027 (2018). arXiv:1803.07989 [hep-ph]

26. E. Eichten, I. Hinchliffe, K.D. Lane, C. Quigg, Super Collider Physics. Rev. Mod. Phys. 56, 579 (1984). https://doi.org/10.1103/ RevModPhys.56.579, https://doi.org/10.1103/RevModPhys.58. 1065 (Addendum: [Rev. Mod. Phys. 58, 1065 (1986)])

27. L. Randall, R. Sundrum, A large mass hierarchy from a small extra dimension. Phys. Rev. Lett. 83, 3370 (1999). https://doi.org/10. 1103/PhysRevLett.83.3370. arXiv:hep-ph/9905221

28. H. Davoudiasl, J.L. Hewett, T.G. Rizzo, Experimental probes of localized gravity: on and off the wall. Phys. Rev. D 63, 075004 (2001). https://doi.org/10.1103/PhysRevD.63.075004. arXiv:hep-ph/0006041

29. K. Lane, S. Mrenna, The Collider phenomenology of technihadrons in the technicolor straw man model. Phys. Rev. D 67, 115011 (2003). https://doi.org/10.1103/PhysRevD.67.115011. arXiv:hep-ph/0210299

30. E. Eichten, K. Lane, Low-scale technicolor at the Tevatron and LHC. Phys. Lett. B 669, 235 (2008). https://doi.org/10.1016/j. physletb.2008.09.047. arXiv:0706.2339 [hep-ph]

31. K. Agashe, R. Contino, A. Pomarol, The minimal composite Higgs model. Nucl. Phys. B 719, 165 (2005). https://doi.org/10.1016/j. nuclphysb.2005.04.035. arXiv:hep-ph/0412089

32. G.F. Giudice, C. Grojean, A. Pomarol, R. Rattazzi, The stronglyinteracting light Higgs. JHEP 0706, 045 (2007). https://doi.org/10. 1088/1126-6708/2007/06/045. arXiv:hep-ph/0703164

33. D. Pappadopulo, A. Thamm, R. Torre, A. Wulzer, Heavy vector triplets: bridging theory and data. JHEP 1409, 060 (2014). https:// doi.org/10.1007/JHEP09(2014)060. arXiv:1402.4431 [hep-ph]

34. H.J. He et al., CERN LHC signatures of new gauge bosons in minimal Higgsless model. Phys. Rev. D 78, 031701 (2008). https:// doi.org/10.1103/PhysRevD.78.031701. arXiv:0708.2588 [hep-ph]

35. T. Aaltonen et al. [CDF Collaboration], Search for $W W$ and $W Z$ resonances decaying to electron, missing $E_{T}$, and two jets in $p \bar{p}$ collisions at $\sqrt{s}=1.96 \mathrm{TeV}$. Phys. Rev. Lett. 104, 241801 (2010). https://doi.org/10.1103/PhysRevLett.104.241801. arXiv:1004.4946 [hep-ex]
36. M. Aaboud et al. [ATLAS Collaboration], Search for diboson resonances with boson-tagged jets in $p p$ collisions at $\sqrt{s}=13 \mathrm{TeV}$ with the ATLAS detector. Phys. Lett. B 777, 91 (2018). https://doi. org/10.1016/j.physletb.2017.12.011. arXiv:1708.04445 [hep-ex]

37. G. Aad et al. [ATLAS Collaboration], Search for diboson resonances in hadronic final states in $139 \mathrm{fb}^{-1}$ of $p p$ collisions at $\sqrt{s}=$ $13 \mathrm{TeV}$ with the ATLAS detector. JHEP 1909, 091 (2019). https:// doi.org/10.1007/JHEP09(2019)091. arXiv:1906.08589 [hep-ex]

38. P. Osland, A.A. Pankov, A.V. Tsytrinov, Probing $Z-Z^{\prime}$ mixing with ATLAS and CMS resonant diboson production data at the LHC at $\sqrt{s}=13$ TeV. Phys. Rev. D 96(5), 055040 (2017). https://doi.org/ 10.1103/PhysRevD.96.055040. arXiv:1707.02717 [hep-ph]

39. I.D. Bobovnikov, P. Osland, A.A. Pankov, Improved constraints on the mixing and mass of $Z^{\prime}$ bosons from resonant diboson searches at the LHC at $\sqrt{s}=13 \mathrm{TeV}$ and predictions for Run II. Phys. Rev. D 98(9), 095029 (2018). https://doi.org/10.1103/PhysRevD. 98.095029. arXiv:1809.08933 [hep-ph]

40. I.A. Serenkova, P. Osland, A.A. Pankov, Improved bounds on WW' mixing with ATLAS resonant WZ production data at the LHC at $\sqrt{s}=13$ TeV. Phys. Rev. D 100(1), 015007 (2019). https://doi. org/10.1103/PhysRevD.100.015007. arXiv:1904.01432 [hep-ph]

41. M. Aaboud et al. [ATLAS Collaboration], Search for $W W / W Z$ resonance production in $\ell v q q$ final states in $p p$ collisions at $\sqrt{s}=$ $13 \mathrm{TeV}$ with the ATLAS detector. JHEP 1803, 042 (2018). https:// doi.org/10.1007/JHEP03(2018)042. arXiv:1710.07235 [hep-ex]

42. A.M. Sirunyan et al. [CMS Collaboration], Search for massive resonances decaying into $W W, W Z, Z Z, q W$, and $q Z$ with dijet final states at $\sqrt{s}=13 \mathrm{TeV}$. Phys. Rev. D 97(7), 072006 (2018). https://doi.org/10.1103/PhysRevD.97.072006. arXiv:1708.05379 [hep-ex]

43. M. Aaboud et al. [ATLAS Collaboration], Searches for heavy diboson resonances in $p p$ collisions at $\sqrt{s}=13 \mathrm{TeV}$ with the ATLAS detector. JHEP 1609, 173 (2016). https://doi.org/10.1007/ JHEP09(2016)173. arXiv:1606.04833 [hep-ex]

44. A.M. Sirunyan et al. [CMS Collaboration], Combination of searches for heavy resonances decaying to $W W, W Z, Z Z, W H$, and $Z H$ boson pairs in proton-proton collisions at $\sqrt{s}=8$ and 13 TeV. Phys. Lett. B 774, 533 (2017). https://doi.org/10.1016/j. physletb.2017.09.083. arXiv:1705.09171 [hep-ex]

45. M.S. Chanowitz, M.K. Gaillard, The TeV physics of strongly interacting $W^{\prime}$ s and $Z^{\prime}$ s. Nucl. Phys. B 261, 379 (1985). https://doi.org/ 10.1016/0550-3213(85)90580-2

46. V. Barger, P. Langacker, H.S. Lee, Six-lepton Z-prime resonance at the LHC. Phys. Rev. Lett. 103, 251802 (2009). https://doi.org/ 10.1103/PhysRevLett.103.251802. arXiv:0909.2641 [hep-ph]

47. V.D. Barger, K. Whisnant, Heavy $Z$ boson decays to two bosons in $E$ (6) superstring models. Phys. Rev. D 36, 3429 (1987). https:// doi.org/10.1103/PhysRevD.36.3429

48. C. Dib, F.J. Gilman, Extra neutral gauge bosons in electron-positron collisions at resonance. Phys. Rev. D 36, 1337 (1987). https://doi. org/10.1103/PhysRevD.36.1337

49. A. Alves, O.J.P. Eboli, D. Goncalves, M.C. Gonzalez-Garcia, J.K. Mizukoshi, Signals for new spin-1 resonances in electroweak gauge boson pair production at the LHC. Phys. Rev. D 80, 073011 (2009). https://doi.org/10.1103/PhysRevD.80.073011. arXiv:0907.2915 [hep-ph]

50. T. Sjostrand et al., An introduction to PYTHIA 8.2. Comput. Phys. Commun. 191, 159 (2015). https://doi.org/10.1016/j.cpc.2015.01. 024. arXiv:1410.3012 [hep-ph]

51. R. Hamberg, W.L. van Neerven, T. Matsuura, A complete calculation of the order $\alpha_{s}^{2}$ correction to the Drell-Yan $K$ factor. Nucl. Phys. B 359, 343 (1991). https://doi.org/ 10.1016/S0550-3213(02)00814-3, https://doi.org/10.1016/ 0550-3213(91)90064-5 (Erratum: [Nucl. Phys. B 644, 403 (2002)]) 
52. Internet https://hepdata.net/

53. S. Frixione, A Next-to-leading order calculation of the crosssection for the production of $W^{+} W^{-}$pairs in hadronic collisions. Nucl. Phys. B 410, 280 (1993). https://doi.org/10.1016/ 0550-3213(93)90435-R

54. N. Agarwal, V. Ravindran, V.K. Tiwari, A. Tripathi, Next-toleading order QCD corrections to $W^{+} W^{-}$production at the $\mathrm{LHC}$ in Randall Sundrum model. Phys. Lett. B 690, 390 (2010). https://doi. org/10.1016/j.physletb.2010.05.063. arXiv:1003.5445 [hep-ph]
55. T. Gehrmann, M. Grazzini, S. Kallweit, P. Maierhöfer, A. von Manteuffel, S. Pozzorini, D. Rathlev, L. Tancredi, $W^{+} W^{-}$production at hadron colliders in next to next to leading order QCD. Phys. Rev. Lett. 113(21), 212001 (2014). https://doi.org/10.1103/ PhysRevLett.136.112001. arXiv:1408.5243 [hep-ph]

56. CMS Collaboration [CMS Collaboration], Combination of diboson resonance searches at 8 and $13 \mathrm{TeV}$. CMS-PAS-B2G-16-007 